\title{
CURRÍCULO Y EDUCACIÓN, SOCIEDAD Y TECNOLOGÍA. IDEAS PARA INICIAR UN DEBATE EN EDUCACIÓN PRIMARIA
}

\author{
Antonio RUEDA MATEOS \\ Víctor AMAR RODRÍGUEZ
}

\begin{abstract}
RESUMEN
El currículo ha de ser un instrumento anclado a la contemporaneidad; de lo contrario estaría obsoleto y caduco. Las coordenadas de espacio y tiempo, vienen unidas a otras de marco tinte social y tecnológico. Hoy en día la sociedad cambia constantemente y el currículo ha de dar respuestas a estas realidades emergidas y emergentes. Pero el matiz tecnológico lo domina de todo, ya que el currículo negado se apodera del proceso y se considera a la corresponsabilidad como un hacedor en el proceso de la Educación Primaria. En el contexto de Andalucía (España) se imparte una asignatura con un carácter marcadamente tecnológico: 'Cultura y práctica digital'. Con su experiencia formativa el alumnado no ha de volver a decir aquello de no lo aprendí porque el maestro no me lo impartió en clase. Ahora hablamos de corresponsabilidad, de curiosidad y motivación como eje motor del proceso y del aprendizaje social en red.
\end{abstract}

PALABRAS CLAVE: Currículo; Sociedad; Tecnología; Cultura digital.

\section{CURRICULUM E EDUCAÇÃO, SOCIEDADE E TECNOLOGIA. IDEIAS PARA INICIAR UM DEBATE NA EDUCAÇÃO PRIMÁRIA}

\section{RESUMO}

O currículo tem que ser um instrumento ancorado na contemporaneidade; do contrário estaria obsoleto e caduco. As coordenadas de espaço e tempo vêm unidas a outras vinculadas ao social e tecnológico. Na atualidade, a sociedade muda constantemente e o currículo deve dar respostas a estas realidades emergidas e emergentes. Mais o matiz tecnológico domina tudo, já que o currículo negado se apodera do processo e se considera à corresponsabilidade como um fato no processo da Educação Primária. No contexto de Andaluzia (Espanha) se ensina uma matéria com um caráter marcadamente tecnológico: 'Cultura e prática digital'. Com sua experiência formativa o aluno não pode dizer que não aprendeu, pois o professor não ensina os conteúdos na aula. Agora falaremos de corresponsabilidade, de curiosidade e motivação como eixo do processo e da aprendizagem social em rede.

PALAVRAS-CHAVE: Curriculum; Sociedade; Tecnologia; Cultura digital.

\footnotetext{
${ }^{\text {i }}$ Maestría en Educación. Formador de ciudadanos del siglo XXI con capacidad para leer contextos locales y globales en respuesta a datos actuales. Me defino como una investigadora y facilitadora del aprendizaje. E-mail: antonio.ruedamateos@gmail.com.

ii Doctorado en Educación. Profesor Titular Universidad de Cadiz-Espanha. E-mail: victor.amar@uca.es.
} 


\title{
CURRICULUM AND EDUCATION, SOCIETY AND TECHNOLOGY. IDEAS FOR INITIATING A DEBATE IN PRIMARY EDUCATION
}

\begin{abstract}
The curriculum must be an instrument anchored to contemporaneity; otherwise it would be obsolete and outdated. The coordinates of space and time are linked to others of a social and technological nature. Today, society is constantly changing and the curriculum must provide responses to these emerging realities. But the technological nuance dominates everything, since the denied curriculum takes over the process and considers co-responsibility as a doer in the process of Primary Education. In the context of Andalusia (Spain) a subject with a markedly technological character is taught: 'Digital Culture and Practice'. With their training experience, students do not have to say that I did not learn it because the teacher did not teach it to me in class. Now we are talking about co-responsibility, curiosity and motivation as the driving force of the process and social learning in the network.
\end{abstract}

KEYWORDS: Curriculum; Society; Technology; Digital culture.

\section{INTRODUCCIÓN A UN DEBATE PROACTIVO}

Los currículos son los caminos del aprendizaje Hans Aebli

Lo habitual ha de ser que el currículo educativo esté en plena concordancia con el momento en el que le ha tocado impartirse. Planteémoslo como lo idóneo. De lo contario, sería un retroceso en la implementación del mismo. Incluso podría ser un caldo de cultivo extraordinario para empezar a hablar de fracaso escolar, algo que determinados profesionales hacen, tal vez, alegremente desde el ámbito de la educación, mientras otros lo cuestionan (PERRENOUD, 2009; SUSINOS, CALVO y ROJAS, 2014). Y está claro que existe fracaso escolar a raíz de la desvinculación del currículo educativo con la sociedad en la que se desenvuelve; sea desde los contenidos y metodología a impartir, hasta la desconsideración del tiempo de atención del alumnado, los recursos, etc. Un vocablo que ya lleva tiempo en los ambientes educativos y se le ha cargado de sentido (RUJAS, 2017).

Pero, igualmente, es evidente que existe fracaso profesional desde el momento en que el docente no reacciona ante el bloqueo o estancamiento de los discentes (DE PUELLES, 2012). El currículo es algo más que unos contenidos que impartir. Es, y simplificando, el hecho concreto por el cual nos vamos a desenvolver; una manera de cualificar a una persona a tenor de un largo proceso que implica desde comprensión de unos contenidos a la capacidad para 
darlo a comprender. Y es cuando se establece la magia recíproca de lo enseñando y de lo aprendido.

Pero, inmediatamente, en este ámbito introductorio la primera pregunta que compartimos con el lector es plantear para qué sirve este currículum educativo (GIMENO 1991; STENHOUSE, 1991). Partiendo de la base de quién lo diseña, empezamos a encontrar respuesta a esta pregunta inicial (CADAVID y CALDERÓN, 2004), pues no debemos olvidar que se atisban concepciones sociológicas e ideológicas, además de otras de ámbito epistemológicas o psicológicas, pedagógicas o didácticas. Y todo conforma lo que se conoce como el currículum oficial.

Incluso, diríamos que está regulado por la ley educativa que establece lo que llamaríamos las enseñanzas. En un macro documento secuenciado y organizado por contenidos temáticos se presenta habilitado para ser impartido $\mathrm{y}$, supuestamente, pensado para un alumnado genérico. La verdad es que topa con la evidencia y, en ocasiones, no se puede impartir según la secuencia impuesta por la oficialidad del documento ni da respuesta a la diversidad del colectivo. De inmediato, nos cuestionamos su valor a modo de para qué sirve. Tal vez, es heredero de parte de un déficit endógeno que lo condena a buscar continuamente mecanismos de mejora pero, del mismo modo, viene avalado por supuestos ecos de garantías de calidad (GOODSON, 2000).

Dicho esto, ya no tenemos muy claro la definición, pues limitarlo a una herramienta pedagógica-didáctica lo convierte en un vehículo para su implementación. O sea, en un instrumento y, de repente, emergen fantasmas que lo identifican con "al servicio de a quién". En su desarrollo muchos currículos educativos se centran en los recursos, contenidos y materiales, se establece una secuencia, quizás, lógica y se determinan los tiempos de ocupación. Pero, ¿nos detenemos a pensar en el componente humano?

Además de aunar, en este discurso que va sucediéndose, que el currículum viene prediseñado desde las autoridades educativas; las cuales pretenden salvar las caracterizaciones propias del centro, la clase o el alumnado con niveles de concreción del mismo. Pero, ya parte de un hándicap. Es elaborado por especialistas, en ocasiones, descontextualizados que permiten, en el mejor de los casos, realizar mejoras sobre lo establecido (PÉREZ GÓMEZ, 2010). Con todo, el currículum oficial cuenta con la estructura esencial que se le permite relativa flexibilidad, incluso se habla de un currículum de aula o de adaptaciones, pero siempre en clara 
relación con una dinámica de asignaturas que enmarcan a los contenidos. Y al hacer algo diferente, se le llama innovación. Pero la propuesta de mejora se establece sobre lo (pre)fijado. El currículo integrado (TORRES, 2006) se aflora como una solución a estas demandas que estamos manifestando.

Ahora bien, la actualidad social, compleja y en continua transformación por los permanentes flujos migratorios, mudanzas económicas y la globalización o los cambios ambientales, científicos y tecnológicos (MONSALVE y AGUASANTA, 2020), demanda un currículo no solo coherente sino, igualmente, positivo e ilusionante, que ilumine la voluntad del alumnado por seguir aprendiendo, encontrándole sentido y proyectándolo en su contexto, en su realidad. Además de incentivar a un docente inspirado que considere que su trabajo tiene sentido proactivo, lejos de quedar como un mero repetidor de contenidos obsoletos y manidos.

No es propio hablar en el currículo por objetivos e, inclusive, de "la obsesión por la eficacia" (GIMENO, 2009). La pedagogía por objetivo fue debate ya hace años, incluso con visos para quedar superada. Se podría pensar en propósitos o finalidades educativas. No son eufemismos que tildan la singularidad para mostrarse diferente. Sino, más bien, una necesidad de empezar a llamar a las cosas y acciones por su nombre. El objetivo está relacionado con el hecho de conseguir ese foco; dar en el blanco. Pero ¿y si no se logra? El proceso, como aquel largo recorrido en compañía de los demás alumnos/alumnas y junto con el docente y la comunidad socioeducativa, se tornan igualmente válidos. La experiencia del aprendizaje no es concluyente sino un proceso que, probablemente, no conozcamos el momento en que se inició ni cuándo concluirá. Ya que, en su comienzo a la experiencia del aprendizaje, el alumnado viene con sus apriorismos y, cuando sale del aula, le queda toda la vida para seguir aprendiendo e, incluso, desaprender y reaprender.

En otro ámbito de cosas, la metodología se esgrime como un instrumento complejo que puede ayudar en el proceso pero, de inmediato, se topa con la evaluación que termina siendo un resorte de calificación que se fundamenta, en ocasiones, en logros o resultados. Olvidándose su posible axioma de conocer para mejorar.

El currículo, por ejemplo, tiene una función facilitadora en el quehacer docente. Es una guía a la que podríamos seguir, máxime cuando se puede llegar a estructurar en un organigrama que se cierra, perfectamente, con la secuencia didáctica. Olvidándose de que, en educación, el trabajar con personas se torna casi imprevisible y, por ello, móvil y dúctil. Mientras que la 
capacidad de adaptación del currículo ha de ser un verdadero resorte para mejorar. La presunción de garantizar una posible homogenización en la propuesta curricular desdice, en cierto modo, su verdadero propósito. Concretarlo puede llegar a ser un verdadero ejercicio de creación y recreación. Obviar la igualdad en las posibilidades (de acceso al sistema educativo) y probabilidades (de salir airoso y con éxito de la experiencia educativa) son acicates y motivos de envite; antes que deseos que se diluyen.

Las personas aprenden constantemente. Los niños, sobre todo, lo hacen de forma excelente. [...].

Las escuelas asumen la responsabilidad de planificar y organizar el aprendizaje de los niños. Intentan -y no con mucho éxito en numerosos casosproporcionar una dirección a dicho aprendizaje y dotarle de un máximo de eficacia (STENHOUSE, 1991, p. 53).

El currículo parte del error que viene con la venia de la formalización. Se concreta casi todo y se deja, en cierto modo, un margen para la maniobra de un contexto que, sin género de dudas, lo explica. En el texto curricular todo - o casi todo- viene especificado; se estipulan los criterios y hasta la fecha de evaluación... Pero, ¿se tiene en cuenta al alumnado en su plenitud? Y no importa que sean pequeños o mayores. No hemos de olvidar que son personas en construcción y lo que hacemos es establecerles pautas y acciones de conocimientos y valores que indican por aquí o por ahí; o mejor dicho: por aquí, pero por ahí no...

Desde la Educación Primaria, el alumnado debe ir creciendo y no solo siendo modelado a imagen y semejanza de la institución. Con la posibilidad de consensuar y teniendo presente el debate de qué aprender, el discente contribuye a su desarrollo personal. Sin embargo, la habilidad del docente debe introducir sugerencias para enseñar en su entorno y en el contexto social al que pertenece. La atención a la diversidad debería ser algo normal en el día a día del aula y no un requisito normativo. La cooperación entre el alumnado no es un mérito, sino una cuestión de cohabitación. El hecho de facilitar los contenidos debe estar inspirado en el respeto a los ritmos de aprendizaje y no a la obediencia o silencio de los estudiantes (CEJUDO et al., 2016).

Para la Educación Primaria, recuperar la tradición de la asamblea o el trabajo por rincones de herencia de la Educación Infantil puede ser una manera de empezar a ver y comprender. Y el trabajo en equipo comenzaría a eclipsar el formato individualizado que se inicia en la tal etapa formativa. Concretar grupos de aprendizaje, dejarlos que se constituyan de 
forma natural según afinidades o establecer estrategias, simples pero idóneas, para promover cambios e intercambios que incentiven dinámicas participativas... Podría ser el comienzo de una buena experiencia formativa y educativa. Incluso, en este quehacer es donde se atisba la singularidad del profesional. Las relaciones entre el alumnado o el diseño de aula son, posiblemente, tan importantes como conocer los ríos o definir el común denominador.

El desarrollo curricular se nos revela como un verdadero escenario de la formación profesional del docente cuando se dan las condiciones necesarias para garantizar la participación del profesorado en el diseño y, sobre todo, cuando la participación se extiende al ámbito de las decisiones para la mejora de la enseñanza y de la escuela (FERNÁNDEZ, 2004, p. 4)

La calidad educativa no solo ha de estar inspirada en el desarrollo del currículo. Es una parte del mismo. La calidad es un conjunto de propiedades que lo define e identifica, haciéndolo específico de una adecuación por y para la educación (GRISAY, 2003), a tenor de la sociedad en que se desenvuelve, los avances científicos y con la impulsión de las tecnologías de la información y la comunicación.

\section{EN EL MARCO DEL ESTUDIO}

No se puede obviar que, en cualquier trabajo de investigación -véase en el contexto educativo-, se hace pertinente contar con un marco contextual. En este caso, adquiere una significación mayor, pues en España la educación está transferida para las diecisiete comunidades autónomas que existen en el estado. Es decir, no se estudia lo mismo ni en la misma lengua en Andalucía, región que servirá de referente, que en Cataluña (en catalán) o Galicia (en gallego). Por ejemplo y en el caso que nos ocupa, la asignatura de 'Cultura y práctica educativa' solo se imparte en esta comunidad del sur de España (que, por cierto, es la más poblada, con casi nueve millones de habitantes). Materia que se encuentra legislada a partir de la Orden de 17 de marzo de 2015 de la Junta de Andalucía.

Cabría añadir que la etapa de Educación Primaria posee el matiz que es obligatoria y gratuita. Y es la que se desarrolla entre la Educación Infantil y la Educación Secundaria Obligatoria (ESO), yendo de seis a doce años. Se estructura en seis niveles (cursos, de primero a sexto) y cuenta con tres ciclos (de dos niveles cada uno). Los pilares sobre los que se asienta esta etapa son: desde la educación comprensiva a la atención a la diversidad, pasando por la 
detección y tratamiento de las posibles dificultades de aprendizaje; con el propósito de contribuir a una educación de calidad inspirada en la formación integral, la equidad y a la adquisición de las competencias clave. Igualmente, juega un papel destacado la atención y acción tutorial, así como la orientación educativa, además de proponerse especial interés en la relación y cooperación con las familias (aspecto en el que hacemos hincapié por determinarlo en plural en relación con la diversidad de modelos de familias que existen). Las familias han de apoyar el proceso educativo (de calidad) de sus hijos.

Y dentro de las diferentes áreas de conocimiento contamos con la cultura digital, que aparece como competencia clave en el diseño de contenidos. Y como referente general cabría señalar la promoción y adquisición de conocimientos, habilidades, destrezas y actitudes con el fin de lograr la realización adecuada de actividades o tareas y la resolución eficaz de problemas complejos en contextos determinados del siglo XXI. Igualmente, cuenta con la orientación metodológica inspirada en el carácter activo, motivador y participativo, nutriéndose de los intereses del alumnado, para favorecer el trabajo individual, cooperativo y el aprendizaje entre iguales.

En cuanto a los contenidos, desde la propia legislación vigente (LOMCE), se especifican que serán:

Bloque 1: "Cultura digital." 1.1. Posibilidades y limitaciones de la sociedad del conocimiento. 1.2. Uso responsable de las redes sociales. 1.3. Concepto de identidad digital. Prevención y actuaciones ante el acoso digital. 1.4. La propiedad intelectual, derechos de autor y creative commons. Bloque 2: "Práctica tecnológica." 2.1. APPs para móviles y tabletas. Aplicaciones de comunicación interpersonal. 2.2. Aplicaciones web 2.0: Comunidades virtuales. Blog. Wikis. Redes sociales 2.3. Utilización de internet para cuestiones cotidianas (recorridos de viajes, consultas de eventos, obtención de entradas a espectáculos, noticias, el tiempo, etc.) Bloque 3: "Educación en línea." 3.1. Los entornos virtuales de aprendizaje: Espacios virtuales, plataformas e-learning. 3.2. Aplicaciones y herramientas Web para el aprendizaje colaborativo. Wikis, repositorios, mensajería, comunidades de aprendizaje. 3.3. Elaboración de blogs y otros recursos web para la comunicación. Mantenimiento de contenidos de plataformas.

Con todo, esta asignatura que obedece al título genérico 'Cultura y Práctica Digital', conforma parte del bloque de asignaturas de libre configuración autonómica, que se nutre con 
la impartida en el quinto curso sobre 'Educación para la Ciudadanía y los Derechos Humanos'. Una experiencia educativa que abraza a lo tecnológico sin soslayarlo de la realidad social y contemporánea.

\section{SOCIEDAD Y CURRÍCULO}

La educación de las personas se establece dentro del marco de la sociedad de referencia. No sería precipitado admitir que, sin sociedad, contexto y momento histórico, no hay currículum; no tendría sentido hacer referencia a ello, pues empezaríamos a balbucear sobre algo irreal y complicado de implementar.

El estudiante se encuentra enmarcado en la sociedad y cultura de referencia (tal vez sinónimo de civilización). Máxime cuando la educación actúa sobre la sociedad, o viceversa, y es la cultura otro elemento de acción sobre la sociedad. Un triunvirato que contribuye al desarrollo social de las personas. Pero debemos establecer un dato más en este incipiente debate; ya que existe una relación entre lo aprehendido en el currículo y el posible éxito social de las personas implicadas en este proceso. En determinado momento, podemos admitir que, en parte, la exclusión social o el determinado fracaso escolar están en este horizonte de partida.

Nos situamos ante un currículo inspirado, fundamentalmente, en los contenidos y, en parte, en la capacidad que tenga el docente para aproximarlos al alumnado y la comprensión de los mismos por parte de los estudiantes. Un ejercicio dual que se establece con un resultado posible que pasa por una preparación activa para dar respuesta a las coordinadas de la vida social e, incluso, política, económica o cultural en que el alumnado se vaya a desarrollar. Entenderá parte de su cotidianeidad y terminará admitiendo, o no, ciertos dictados establecidos como beneficiosos, además de poder llegar a ser un elemento de obediencia o de rechazo; una forma de empezar a determinar lo que se considera el bien o el mal. Con ello, a través de la transmisión de los contenidos curriculares, el alumnado abre la caja de pandora de su preparación para la vida en sociedad. En ocasiones, se le incentiva el espíritu crítico; pero, en otros tantos momentos de este quehacer, la rutina se abre paso y se asienta sobre el procedimiento. La propuesta activa se diluye entre un marasmo de contenidos curriculares que imposibilitan, en parte, esta propuesta de cambio (GIMENO, 2018). 
Probablemente, el llamamiento para que el currículum sea un hecho preparatorio para la vida en sociedad y social comienza a disolverse. La función de la escuela es otra, la cual quizás- presenta su matiz más continuista e inamovible. La promoción escolar se inspira en la capacidad de transmitir los contenidos curriculares fundamentados en la formación reglada. Lejos comienzan a quedar aquellos valores o matices sociales que posibilitarían, no sé sabe en qué grado, el éxito social o profesional; pero, probablemente, se torna en un vehículo de superación personal, incluyéndose desde la resiliencia a la compatibilidad o rechazo de lo establecido consuetudinariamente. Saber diferenciar entre doctrina y currículo se hace necesario, lo cual es reflejado por DE LA HERRÁN (2012, p. 287) de la siguiente manera:

El criterio para diferenciar lo doctrinario de lo curricular será precisamente considerar 'curricular' a lo que favorece la formación y la autoformación, esto es: lo que desde la cultura no fomenta la parcialidad, la identificación, la dualidad y la fragmentación humana, y en cambio profundiza y amplía la conciencia, desarrolla la complementariedad, promueve la universalidad, el descondicionamiento, pretende la autonomía y el conocimiento de la persona, etc.

Pero la pregunta que compartimos con el lector en este marco de relaciones entre la sociedad y el currículo es: ¿Quién se implica en este posible cambio de estrategia y de resultados curriculares en relación con lo social?

La institución tiene sus empeños; el docente su compromiso; pero el alumnado, si no empieza a permitírsele tomar iniciativas desde su infancia, se termina acostumbrado a la rutina curricular preestablecida, extrayendo rédito y acomodándose para no tener conflictos. En definitiva, para promocionar de curso.

Aquel principio sobre el que asentamos la función curricular de ser un útil para la preparación y toma de consciencia en la actividad social, se torna una distopía. La vida social pasa de largo por el currículo oficial y puede aparecer, esporádicamente, en el currículo oculto (el de las intenciones). Bien intencionado, el currículo oculto conforma parte de las mejores miras de un docente que, implicado, quiere que su alumnado sea una buena persona, de éxito y feliz en la sociedad que le ha tocado vivir. Pero, a la vez, que pueda entender la vida para no sufrir más de la cuenta y, si es posible, modificarla en la medida de sus capacidades y responsabilidades. Y es cuando comienza a dar sus frutos aquella parte invisible del currículo impartido por el docente que se posicionaba, con la intención de presentarse como un espejo 
ante sus alumnos, para que ellos adviertan la posibilidad y necesidad crítica del currículo en la sociedad de su tiempo, además de comprender otros comportamientos; sin tener que necesariamente terminar admitiéndolo. A grosso modo, se comienza a suscribir aquel necesario impulso crítico (DEL BASTO y OVALLE, 2015).

Pero el siguiente paso en este currículo optimista (TORRES, 2001, p. 65) se establece en base a "una escuela en la que el alumnado se sienta animado a vivir e intervenir con libertad y optimista en la sociedad, una escuela, en definitiva, generadora de sueños y no de sueño".

Ya no vale solo con una propuesta teórica; hace falta un paso hacia delante, cualitativo, que pasa por la acción práctica: la posibilidad de implicarse en un cambio social, entiéndase en un sentido procomunitario. Estableciéndose, a modo de ejemplo, por las relaciones de género, la igualdad, la corresponsabilidad en el hogar, lo ambiental, lo cívico y la ciudadanía, entre otros asuntos. A la postre, son aspectos curriculares que tienen un flash en los contenidos pero que no conforman parte del entramado curricular oficial. Una percepción sesgada lo hace tan engañosa como incompleta. Por ello, la labor curricular pasaría, igualmente, por visibilizarlos y darlos a comprender. Estamos proponiendo el fin de aquel currículo sesgado que no mira a lo social y a la sociedad. Su comprensión se centra en su puesta en valor y práctica en torno al devenir del alumnado. La función social de la escuela se evidencia, más si cabe, a partir de las palabras de GIMENO (1991, p. 18), pues fue "configurada para desarrollar el proceso de socialización de las nuevas generaciones y que garantiza la reproducción social y cultural como requisito para la supervivencia misma de las sociedades".

Y para abordar esta naturaleza inamovible y sesgada del currículo hace falta reformular la formación del docente (BOLÍVAR, 2004). En este sentido, abogamos por un currículo reescrito en tiempo presente, revisado y revisionista que se centre en la consideración e importancia que tiene y mantiene el conocimiento y reconocimiento del mundo y las relaciones sociales que nos ocupan y preocupan.

Un currículo se nutre de las páginas de los libros de texto y, también, abriendo las aulas para permitir la entrada de lo social: desde una asociación de emigrantes o una propuesta de visita a una fábrica. No se ha de limitar a lo preestablecido en exclusividad, por aquello que viene dado en el currículum oficial y determinado con anterioridad. Precisamos de un ejercicio de apertura de miras donde otear la sociedad; que, al fin y al cabo, es nuestro marco de referencia y desarrollo, en el amplio sentido del término. 
Con ello, realizamos un llamamiento a la inclusión de dinámicas de aula más abiertas que se nutran con la visita de personas externas al centro, con familiares de alumnado, con miembros especialistas del Ayuntamiento o de algún colectivo, etc. Y que sea el motivo que incentive un trabajo por proyecto que deje la semilla que pueda llegar a germinar en el vacío de la sensibilidad del alumnado, preocupado por las áreas de conocimiento sin, apenas, mirar a la cara del compañero que tiene a su lado, sin reparar en lo prosocial, sin considerar que algo podríamos aportar para mejorar la vida o, al menos, la cotidianeidad. O que la vida no nos cambie demasiado... Teniendo como aliado al diálogo, el análisis, la reflexión pero, igualmente, la crítica y la autocrítica.

En cierto modo, hemos pretendido presentar y colocar en el debate la necesidad de un currículo activo e ilusionado, no solo ambientado en los contenidos, sino en las cuestiones de la sociedad, verdadero basamento de acción y actuación. Un currículo en conexión con lo social $\mathrm{y}$, sobre todo, con las personas y problemáticas que conforman parte del siglo XXI (CARBONELL, 2015). A la postre, estamos compartiendo la preocupación dual de que existen unos conocimientos, versionados en lo educativo como contenidos sociales, que están silenciados u omitidos, además de que en la actualidad, con el advenimiento y desarrollo de las tecnologías de la información y la comunicación, cada vez está más complicado separar entre un saber social de otro que sea considerado, exclusivamente, escolar. Los vasos comunicantes y comunicativos son, extraordinariamente, lúcidos; pues las pantallas realizan un papel catalizador de mostrar aquella realidad que el alumnado desea ver, aprender y compartir.

\section{CURRÍCULUM $\quad$ Y TECNOLOGÍAS DE LA INFORMACIÓN $\quad$ Y LA COMUNICACIÓN}

A priori, puede llegar a ser algo precipitado establecer una relación entre el currículum y las tecnologías de la información y la comunicación. No debemos obviar que se trata de un ámbito de la educación que se corresponde con el nivel de primaria. En el contexto español, e insistimos en ello, se imparte con alumnos y alumnas de entre seis y doce años de edad. Lo que significa que, tal vez, no estén demasiado duchos para desenvolverse entre las tecnologías; pero sí para desarrollar un matiz personal y educativo que pasa por la corresponsabilidad. Es decir, somos conscientes de la poca edad y grado madurativo de este alumnado de primaria. No 
obstante, desde pequeños se les ha de ir inculcando que lo que explica el docente en el aula o lo que aparece en los libros de textos son un pretexto para aprender aquello focalizado. Todavía les queda mucho por seguir aprendiendo. Máxime cuando existe una asignatura concreta en sexto de primaria que obedece al título de 'Cultura y práctica digital', al menos en el contexto de Andalucía que, como ya dijimos, tiene transferida las competencias educativas y, en parte, curriculares.

El alumnado viene vinculado a las tecnologías desde su propia cotidianeidad. En el aula continúa con la adquisición de conocimientos. Puede aprender desde cualquier dispositivo inalámbrico y se produce un modo de aprender social que hasta el momento no tenía precedente (BURBULES, 2014; SANTOS, GONZÁLEZ y MUÑOZ, 2018; CÁRDENAS y CÁCERES, 2019). En las aulas existen pizarras electrónicas, además de las posibles tabletas de las que el alumnado pueda llegar a disponer. Pero, sea como fuera, una vez el discente llega a sexto de primaria, cambia la semántica del currículo, pues se encuentra con unos contenidos que impulsan y deconstruyen parte de lo que aprende, además de cómo, cuándo y con quién lo aprende.

El alumnado debe estar incentivado en la manera de buscar la información. En un mar de datos no se le puede permitir naufragar (sinónimo de distracción, pérdida o recorrido sin fundamento). Desde enseñarles a buscar en buscadores o metabuscadores hasta restringir la búsqueda puede ser un buen comienzo. Una vez se alcanza la orilla del conocimiento se le puede empezar a encaminar sobre la selección de la información encontrada. En este sentido, tanto se peca por defecto como por exceso. Es ahí donde la mesura y experiencia del docente debe establecer pautas para consensuar criterios de selección y que sea el primer paso para la evaluación de los contenidos encontrados. Una vez se dispone de la información, se ha de pasar a la cualificación de la misma, sea a través de un ejercicio de constatación y de actualización de ella. Dos coordenadas que reubicarán la navegación del alumnado.

Por último, se ha de saber compartir. Es imprescindible ser generoso; se trata de un valor universal y educativo. Es una manera de dar a comprender, donde se genera una valía en el estudiante que lo promueve. En este sentido, el hecho de compartirlo se convierte en un aliado de la nueva manera de evaluar que no solo se ha de llevar a cabo por lo mucho o poco que el alumnado pueda atesorar, sino también por la manera que ha tenido de dar a comprender, compartiendo su conocimiento con sus compañeros. 
Y al estimado currículo se le aparece una fuga de agua que hace que el barco, en vez de hundirse en este ejercicio de navegación, una vez subsanado el cascarón, se refuerce. El saber está ahí y el alumnado tiene herramientas para buscar y comprender, además de capacidad para hacerlo suyo y compartirlo. No es dependiente de los dictados (a veces, caprichos) de los docentes o del currículo. Y, añadido al conocido como currículo oficial (el dado por la institución educativa), real (el impartido verdaderamente en el contexto de aula) y oculto (incluyéndose desde valores y normas que no aparecen reconocidos), emergen otros dos a modo de referente: el currículo negado (como aquel que no se imparte por razones de tiempo, desconocimiento, etc.) y el que hace alusión al currículo nulo (semejante al negado, que matiza su rechazo por llegarse a considerar repleto de contenidos superfluos e innecesarios o, incluso, inútiles) (ARRIETA y MESA, 2001). Estamos ante un ejercicio de acercamiento y análisis del currículo (POSNER, 2004).

Ahora bien, cabe cuestionarse qué podemos hacer si en la red está la información presentada de modo internivelar, con capacitación didáctica y respetando el ritmo de aprendizaje del alumnado, además de gamificada o interactiva (CARRERAS, 2016). El papel que juega el currículo negado se dimensiona. Es más, el alumnado se verá cuestionado para afirmar que ese determinado contenido no lo conoce o no lo sabe, pues no lo han dado en su clase. A partir de este momento, el docente se arma de la corresponsabilidad como aliada para impulsar el saber. Y el alumnado tiene a la corresponsabilidad como artilugio para decir que, si no lo sabe, lo puede buscar y conocer. Y la motivación se erige como referente para promover el conocimiento.

Y desde este momento las tecnologías revolucionan el currículum. Se convierten en aliadas que funcionan como espejo del saber del aula y hacen que el alumnado no deje de ocultar la necesidad de saber. La escuela, como ayer, se ve modificada para atisbarla como un presente con futuro. No significa soslayar las responsabilidades de unos y otros. A partir de ahora, tanto docentes como discentes se irán a desenvolver en el mar de la corresponsabilidad.

\section{CONSIDERACIONES FINALES}

Con la intención de impulsar más si cabe el debate abierto sobre la corresponsabilidad, las tecnologías de la información y la comunicación, así como con el matiz impuesto por la 
corresponsabildidad curricular en el aprendizaje, pretendemos ir avanzando con un nuevo interrogante. En este sentido, la siguiente cuestión que proponemos es: ¿Y si nuestra educación estuviese radicalmente equivocada? (DE LA HERRÁN, 2017).

En cierto modo, no encontramos una respuesta a esta pregunta. Tan solo la colocamos para proseguir el debate iniciado desde párrafos anteriores. Sin duda se han abierto caminos y derivas (SANCHO et al., 2020). El derrotero de la navegación viene dado por los indicadores de brújula y sextantes que muestren una aproximación a puertos seguros. La figura del docente se redimensiona en vez de creer que se amilana o minimiza ante el envite de las tecnologías. Es más, diríamos que se incrementa desde el momento en que las tecnologías de la información y la comunicación (TIC) se hacen tecnologías de las relaciones, de la información y la comunicación (TRIC) (MARTA-LAZO y GABELAS, 2016). Y se redimensiona el matiz de las relaciones como acicate para desenvolver las capacidades de aprender y compartir en alumnado y profesorado.

'Currículo y educación, sociedad y tecnología. Ideas para iniciar un debate en Educación Primaria', no deja de ser un pretexto para reflexionar en voz alta sobre lo que se avecina en tiempos de cambios. Son retos impuestos por la contemporaneidad que obligan a una reacción del sistema educativo que está enmarcado en las coordenadas de la sociedad y la tecnología. Una manera nueva de leer, que pasa de lo lineal del papel a lo concéntrico de las pantallas. Un modo singular de aprender, donde la socialización se plantea entre muchos, más allá de la propia realidad del aula para hacerse extensa y adquiriendo el matiz de aprendizaje social en red (GARCÍA ARETIO, 2019). Una forma particular de combinar lo que se aprende con las realidades de unas tecnologías para la transformación de la educación (PEDRÓ, 2017).

Una fusión que produce un resultado inexacto en pro de una mezcolanza establecida entre la tecnología y el conocimiento, en unión con el currículo. Un currículo que ha dejado de ser estático y estar en las manos y en las mentes de los responsables educativos para optar a ser patrimonio de otros y estar extendido. Ahora bien, no con la condición de dejar solo al alumnado, sino de formarles y ayudarles a crecer como personas del y para el siglo XXI.

Por último, cabría añadir que la capacidad de compartir el conocimiento del alumnado en unión con el desarrollo de las TIC y las TRIC otorga una redimensión al currículo. Tal vez, un desarrollo parecido al experimentado, ya hace años, por el currículo oculto (TORRES, 1996) en la cotidianeidad del aula y que tanto bien ha aportado. 
Asimismo, la evaluación tradicional ha dejado de ser el espacio único para estimar los conocimientos y rendimientos del alumnado, para llegar a ser un entorno para compartir el conocimiento, ampliando el provecho y las habilidades de los implicados en el proceso de enseñanza-aprendizaje. El nivel de conocimiento del alumnado no solo se mide desde el saber acumulado, sino también desde la capacidad de éxito y la facultad para dar a comprender lo aprendido, compartiéndolo en la red. Y seguimos con el debate a partir de:

La profesión docente está íntimamente ligada a la evaluación. La realizamos constantemente siguiendo unos criterios preestablecidos, pero ¿evaluamos bien?, ¿adoptamos criterios correctos?, ¿evaluamos cómo nos evaluaron a nosotros? Evaluar con intención formativa no es lo mismo que calificar, medir o corregir; examinar, clasificar o aplicar pruebas objetivas (GRAU y GÓMEZ, 2010, p. 18).

Una evaluación en consonancia con las tecnologías. Pues la nueva escritura o lectura y los novedosos modos y cualidades de aprender se han de evaluar también. No vale mantener el pasado inmóvil. Hace falta abrazar las realidades tecnológicas y sociales en la educación.

En definitiva, el currículo lo establecemos como una concreción específica que obedece a un proyecto político y educativo, en clara respuesta a un modelo pedagógico social (que hacemos extensible a lo tecnológico). En él se dan cita desde la propia pedagogía con su visión sobre los planes de estudios hasta el matiz curricular como pilar donde se asienta el proceso de enseñanza-aprendizaje y, además, señalar la vertiente didáctica como el arte de enseñar todo a todo el mundo atendiéndose a los ritmos del alumnado. Sería algo parecido a una versión microcurricular interpretada y desarrollada en la unidad didáctica. Una tradición empeñada en “comprender y transformar la enseñanza” (GIMENO y PÉREZ, 1995).

Estamos convencidos de que el recorrido entre las tecnologías, lo social y la educación va a tener un largo trayecto. Máxime cuando los hechos históricos, en clara coherencia con lo económico, sanitario o político, nos obligan a adaptarnos a las nuevas realidades que se inspiran en la educación virtual e, igualmente, en la reconsideración del currículo y su innovación (CASANOVA, 2009).

En todo caso, hemos determinado una sutil diferencia entre el currículum negado, como aquel que no se imparte, y el nulo, como el que se considera con escasa aplicabilidad o aparente utilidad, que puede llegar a ser tildado en cuanto a contenidos de superfluo. Lejos queda el currículo extraescolar; con una evidente pretensión voluntaria y recreativa, llevándose a cabo 
en horario no lectivo y no siendo evaluable. Algo que no se ha de confundir con las actividades complementarias, enmarcadas en lo curricular, en horario lectivo, evaluable y obligatoria. Y todos los modelos de currículo, en su mayor o menor medida, contribuyen al desarrollo integral del estudiante.

\section{REFERÊNCIAS}

ARRIETA, Beatriz; MESA, Rafael. El currículum nulo y sus diferentes modalidades. Revista de Educación, 25(1); p. 1-9, 2001. Doi https://doi.org/10.35362/rie2513143. Acceso el: 28 jul; 2020.

BOLÍVAR, Antonio. Formación permanente del profesorado y desarrollo del currículum. Revista Colombiana de Educación, 47, p. 57-73, 2004. Recuperado de: https://revistas.pedagogica.edu.co/index.php/RCE/article/view/5513/4540. Acceso el: 30 jul. 2020.

BURBULES, Nicholas. El aprendizaje ubicuo: nuevos contextos, nuevos procesos. Entramados: educación y sociedad, 1(1), p. 131134, 2014. Recuperado de: http://fh.mdp.edu.ar/revistas/index.php/entramados/article/view/1084. Acceso el: 4 ago. 2020.

CADAVID, Ana; CALDERÓN, Isabel. Análisis del concepto enseñanza en las teorías curriculares de Lawrence Stenhouse y José Gimeno Sacristán. Revista Educación y Pedagogía, XVI(40), (septiembre-diciembre), 2004, p. 143-152.

CARBONELL, Jaume. Pedagogías del siglo XXI. Alternativas para la innovación educativa. Barcelona: Octaedro, 2015.

CÁRDENAS, Irma; CÁCERES, Maritza. Las generaciones digitales y las aplicaciones móviles como refuerzo educativo. Revista Metropolitana de Ciencias Aplicadas, 2(1), p. 25-31, 2019. Recuperado de http://remca.umet.edu.ec/index.php/REMCA. Acceso el: 3 ago. 2020.

CARRERAS, Carla. Del homo ludens a la gamificación. Quaderns de Filosofia, 4(1), p. 107-118, 2017. doi: 10.7203/qfia.4.1.9461. Acceso el: 28 jul. 2020.

CASANOVA, María. Diseño curricular e innovación educativa. Madrid: La Muralla, 2009. CEJUDO, Javier et al. Necesidades de formación de maestros de infantil y primaria en atención a la diversidad. Bordón. Revista de Pedagogía, 68(3); p. 23-39, 2016.

DEL BASTO, Liliana; OVALLE, María. Una mirada crítica a la relación currículo sociedad. Revista Latinoamericana de Estudios Educativos, 1(11), p. 111-127, 2015. Recuperado de: http://190.15.17.25/1atinoamericana/downloads/Latinoamericana11(1)_7.pdf. Acceso el: 2 ago. 2020. 
DE LA HERRÁN, Agustín. Currículo y pedagogías renovadoras en la edad antigua. REICE Revista Iberoamericana sobre Calidad, Eficacia y Cambio Educativo, 10(4), p. 285-334, 2012. Recuperado de: http://www.rinace.net/reice/numeros/arts/vol10num4/art17.pdf. Acceso el: 26 jul. 2020

DE LA HERRÁN, Agustín. ¿Y si nuestra educación estuviese radicalmente equivocada? In: MEDINA, Antonio; DE LA HERRÁN, Agustín y DOMÍNGUEZ, María (Coords.). Nuevas perspectivas en la formación de profesores. Madrid: Universidad de Educación a Distancia (UNED), 2017. p. 457-517.

DE PUELLES, Manuel (Ed.). Fracaso escolar en el estado de las autonomías. Madrid: Wolters Kluwer, 2012.

FERNÁNDEZ, Manuel. El desarrollo docente en los escenarios del currículum y la organización. Profesorado. Revista de currículum y formación del profesorado, 8(1), 2004, p. 1-20. Recuperado de: https://www.ugr.es/ recfpro/rev81COL3.pdf. Acceso el: 1 ago. 2020.

García Aretio, Lorenzo. Necesidad de una educación digital en un mundo digital. RIED.

Revista Iberoamericana de Educación a Distancia, 22(2), p. 9-22, 2019. doi:

http://dx.doi.org/10.5944/ried.22.2.23911. Acceso el: 2 ago. 2020.

GIMENO, José. El Curriculum: Una Reflexión sobre la Práctica. Madrid: Morata, 1991.

GIMENO, José. La pedagogía por objetivos: obsesión por la eficiencia. 11. ed. Madrid: Morata, 2009.

GIMENO, José. Cambiar los contenidos, cambiar la educación. Madrid: Morata, 2018.

GIMENO, José; PÉREZ, Ángel. Comprender y transformar la enseñanza. Madrid: Morata, 1995.

GOODSON, Ivor. El cambio en el currículum. Barcelona: Octaedro, 2000.

GRAU, Salvador; GÓMEZ, María. La evaluación, un proceso de cambio para el aprendizaje. In: GÓMEZ, María; GRAU, Salvador (Coords.). Evaluación de los aprendizajes en el espacio europeo de educación superior. Alicante: Universidad de Alicante, 2010. p. 17-32.

GRISAY, Aletta. Repetir curso o adecuar el currículum. In: MARCHESI, Álvaro y HERNÁNDEZ, Carlos (Coords.). EI fracaso escolar: una perspectiva internacional. Madrid: Alianza, 2003. p. 101-128.

JUNTA DE ANDALUCÍA. Orden de 17 de marzo de 2015. Recuperado de: https://www.juntadeandalucia.es/boja/2015/60/1. Acceso el: 4 ago. 2020. 
MARTA-LAZO, Carmen; GABELAS, José. Comunicación digital. Un modelo basado en el factor r-elacional. Barcelona: UOC, 2016.

MONSALVE, Laura; AGUASANTA, Miriam. Nuevas ecologías del aprendizaje en el currículo: la era digital en la escuela. Revista Latinoamericana de Tecnología Educativa, 19(1), 2020. Doi: https://doi.org/10.17398/1695-288X.19.1.139. Acceso el: 2 ago. 2020.

PÉREZ GÓMEZ, Ángel. Reinventar la profesión docente. Nuevas exigencias y escenarios en la era de la información y la incertidumbre. Revista Interuniversitaria de Formación del Profesorado, 68, p. 17-36, 2010. Recuperado de:

https://www.redalyc.org/pdf/274/27419198002.pdf. Acceso el: 29 jul. 2020.

PERRENOUD, Philippe. La construcción del éxito y del fracaso escolar. Madrid: Morata, 2008.

POSNER, Georges. Análisis del Currículo. 4. ed. México: Mc Graw Hill, 2004.

RURJAS, Javier. La construcción del «fracaso escolar» en España. Génesis y cristalización de un problema social. Papers, 102(3), 477-507, 2017. doi:

http://dx.doi.org/10.5565/rev/papers.2297. Acceso el: 3 ago. 2020.

SANCHO, Juana et al. (Coords.). Caminos y derivas para otra investigación educativa y social. Barcelona: Octaedro, 2020.

SANTOS, Francisco; GONZÁLEZ, Mercerdes; MUÑOZ, Pablo. El desarrollo de las ecologías de aprendizaje a través de las herramientas en línea. Revista Diálogo Educacional, 18(56), p. 128-148, 2018. doi: 10.7213/1981-416X.18.056.DS06. Acceso el: 29 jul. 2020

STENHOUSE, Lawrence. Investigación y Desarrollo del Curriculum. 3. ed. Madrid: Morata, 1991.

SUSINOS, Teresa; CALVO, Adelina; ROJAS, Susana. El fracaso escolar y la mejora de la escuela. Madrid: Síntesis, 2014.

TORRES, Jurjo. El currículo oculto. Madrid: Morata, 1996.

TORRES, Jurjo. Un currículo optimista fronte á desmemoria e o fatalismo. Lección inaugural en la solemne apertura del curso 2001-2002 en la Universidad de La Coruña. La Coruña: Universidad de La Coruña. 2001.

TORRES, Jurjo. Globalización e interdisciplinariedad: el currículum integrado. Madrid: Morata, 2006. 
Recebido em: 07/08/2020

Aprovado em: 09/11/2020 